\title{
Green synthesis and charaterization of magnetic nanoparticles for electrocatalysis
}

\author{
Harsha Devnani*, Sana Ansari and Soami P. Satsangee \\ University Science Instrumentation Centre, \\ Dayalbagh Educational Institute, Dayalbagh, Agra-282005 (India) \\ *Email: harsha.devnani@gmail.com
}

Nanomaterials exhibit several intriguing properties such as an enhanced surface area to volume ratio, high surface reactivity and catalytic efficiency, which makes them a potential candidate for the fabrication of various sensors. The synthesis of magnetic nanoparticles has attracted much attention owing to their immense potential for various applications such as advanced magnetic materials, high-density magnetic recording media, medical diagnostics and catalysis [1,2]. Magnetite is the most common magnetic iron oxide having a cubic inverse spinel structure which shows interesting electric and magnetic properties due to the transfer of electrons between the ferrous and ferric ions in the octahedral sites [3]. There are a large number of physical, chemical, biological and hybrid methods available to synthesize nanoparticles but green nanotechnology has gained impetus to reduce the amount of toxic chemicals in the environment [4]. The phytochemicals present in the plant extracts act as reducing as well as capping agents and thus act as building blocks for the formation of nanoparticles. In the present work, the magnetic nanoparticles were synthesized using an aqueous extract of Vetiver roots without the intervention of any man-made chemicals. The ferric chloride salt was mixed with the aqueous extract of vetiver roots in a 1:1 ratio and stirred for an hour. The bown-black color indicated the formation of magnetic nanoparticles (MNPs). They were characterized using UV-VIS spectroscopy, which showed absorbance maxima at $430 \mathrm{~nm}$ (Figure 1).

The surface morphology was analyzed using scanning electron microscopy (SEM). Figure 2 shows the structure to be flaky with ridges thereby indicating a rough surface.

The electroctalytic activity of the synthesized MNPs was checked by performing cyclic voltammetry in 1 $\mathrm{mM}$ ferricyanide system at the bare carbon paste electrode (CPE) and at MNP modified CPE (MNP/CPE) (Figure 3)

The enhanced peak currents were obtained at $\mathrm{MNP} / \mathrm{CPE}$ in comparison to bare CPE and also the oxidation potential shifted to negative values indicating the electrocatalytic role of MNPs. The MNPs thus synthesized by green route can be effectively used for electrocatalytic application.

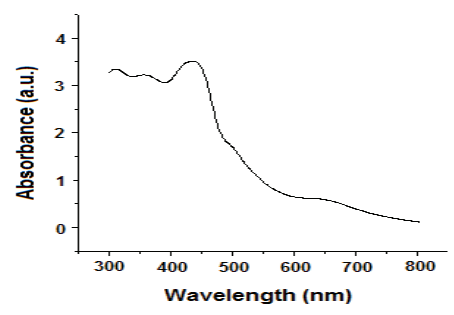

Figure 1: UV-VIS spectra of MNPs

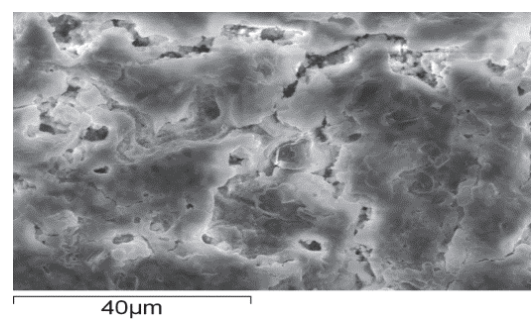

Figure 2: SEM image of MNP

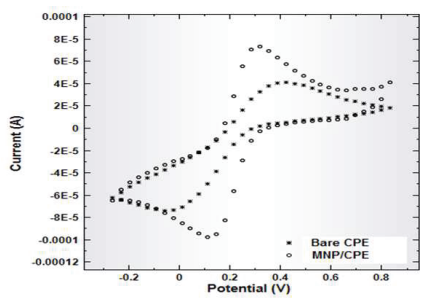

Figure 3: Cyclic volatmmograms in $1 \mathrm{mM}$ ferricyanide solution at bare and modified electrode

\section{References}

1. C. Prasad, S. Gangadhara and P. Venkateswarlu. Appl. Nanosci. 2015,doi:10.1007/s13204-015-0485-8

2. S. Saha and A.K. Bhunia. J. Phys. Sci. Vol.17, pp.191195, 2013.

3. Abhilash, K. Revati and B.D. Pandey. Bull. Mater. Sci. Vol.34, pp.191-198, 2011.

4. M. Mahdavi, F. Namvar, M.B. Ahmad and R. Mohamad. Molecules Vol.18, pp.5954-5964, 2013. 